\title{
CP violation in top quark decay via heavy Majorana neutrinos at the LHC
}

\author{
Peng-Cheng Lu $\odot,{ }^{1, *}$ Zong-Guo Si, ${ }^{1, \dagger}$ Zhe Wang $\odot,{ }^{1, *}$ and Xing-Hua Yang $\odot^{2, \S}$ \\ ${ }^{1}$ School of Physics, Shandong University, Jinan, Shandong 250100, China \\ ${ }^{2}$ School of Physics and Optoelectronic Engineering, Shandong University of Technology, \\ Zibo, Shandong 255000, China
}

(Received 22 October 2021; accepted 14 November 2021; published 6 December 2021)

\begin{abstract}
The tiny neutrino masses can be explained naturally by extending the standard model with right-handed Majorana neutrinos in a low-scale seesaw mechanism, and the $C P$ violation effect induced by the Majorana phase is interesting and worth studying at colliders. In this paper, we investigate the prospects for measuring $C P$ violation in top quark pair production and rare decay via Majorana neutrinos at the LHC. The $C P$ asymmetry stems from the significant interference of contributions from two different Majorana neutrinos and can be appreciable when the two neutrino masses are nearly degenerate. It is found that in the Majorana neutrino mass range of $10 \mathrm{GeV}<m_{N}<80 \mathrm{GeV}$, the $C P$ asymmetry is independent of the Majorana neutrino mass at the LHC. Any possible new observation of $C P$ violation will be the clear evidence of new physics beyond the standard model.
\end{abstract}

DOI: 10.1103/PhysRevD.104.115003

\section{INTRODUCTION}

The present evidence of neutrino masses and mixings implies the existence of new physics beyond the standard model [1]. To generate the tiny neutrino masses, the simplest way is to extend the standard model by introducing $n$ righthanded Majorana neutrinos $N_{a R}(a=1,2, \ldots, n)$. Since only two active neutrino mass-squared differences have been measured experimentally, the number of right-handed neutrinos is limited to $n \geq 2$. With right-handed Majorana neutrinos, the Dirac neutrino mass terms can be generated through Yukawa interaction after spontaneous gauge symmetry breaking, just like other standard model fermions. In addition, the introduced Majorana neutrinos and their charge-conjugate counterparts can also form Majorana mass terms as they are $S U(2)_{L}$ gauge singlets. This is known as the famous type I seesaw mechanism [2-6], where the light neutrino masses are inversely proportional to the large mass scale of Majorana neutrinos. Unfortunately, in the canonical high-scale type I seesaw mechanism, the heavy Majorana neutrinos are too heavy, and their mixing with active neutrinos is too weak for them to be observed at current and future experiments. However, there also exists some

\footnotetext{
pclu@sdu.edu.cn

zgsi@sdu.edu.cn

*whe@mail.sdu.edu.cn

§yangxinghua@sdut.edu.cn
}

Published by the American Physical Society under the terms of the Creative Commons Attribution 4.0 International license. Further distribution of this work must maintain attribution to the author(s) and the published article's title, journal citation, and DOI. Funded by SCOAP ${ }^{3}$. low-scale type I seesaw mechanisms with the light neutrino masses being directly proportional to a small leptonnumber breaking scale. Two of the most popular scenarios are inverse seesaw [7-10] and linear seesaw [11,12]. Moreover, the right-handed neutrinos with masses below the electroweak scale could also explain the baryon asymmetry of the Universe via leptogenesis [13] and be a natural dark matter candidate [14-17]. From the experimental point of view, we will focus on the low-scale seesaw scenarios.

The existence of Majorana neutrinos will at the same time lead to the violation of the lepton number $(\Delta L=2)$, which provides a unique opportunity to search for the Majorana neutrinos via lepton-number-violating processes. For example, the widely studied processes in low energy regions include the well-known neutrinoless double beta decays $(0 \nu \beta \beta)$ [18-20], the rare meson decays [21-24], and the tau lepton decays [25-27]. In addition, the same signals can also be searched for at various collider experiments [28-39]. The key point of the lepton-number-violating processes mentioned above is a $W$ decay via Majorana neutrino exchange, which can be specifically expressed as $W^{-} \rightarrow \ell_{1}^{-} \ell_{2}^{-}\left(q^{\prime} \bar{q}\right)^{+}$. Generally, both the light Majorana neutrinos and the heavy Majorana neutrinos can contribute to the $W$ decay amplitude. However, the light Majorana neutrino contributions are strongly suppressed and can be safely neglected due to their small neutrino masses, which are at most $\mathcal{O}(\mathrm{eV})$ [32]. The $C P$ violation effect will be the smoking gun for new physics beyond the standard model. If more than one heavy Majorana neutrino participates in the lepton-number-violating $W$ decay, then it may be possible to produce new sources of $C P$ violation, which can play an important role in explaining the baryon asymmetry of the 
Universe. To be specific, the $C P$ asymmetry can be generated from the significant interference of contributions from different Majorana neutrinos. There have been a great number of theoretical works on examining the prospects for observing $C P$ violation via lepton-number-violating processes, where the $C P$ violation can be measured in the decays of mesons [40-47] and tau leptons [48,49]. Recently, the possibility for measuring $C P$ violation in $W^{+}$and $W^{-}$decay at the LHC was explored [50]. However, the $C P$ violation effect is influenced by the initial parton distribution functions (PDF), which leads to the different production cross section of $W^{+}$and $W^{-}$. To avoid the pollution from PDF, in this paper, we investigate the prospects for measuring $C P$ violation in top quark pair production and rare decay at the $\mathrm{LHC}$, where the number of $W^{-}$and $W^{+}$bosons coming from the decay of $\bar{t}$ and $t$ is exactly equal. Since the LHC is a top rich environment, this provides a great opportunity for using the copious $t \bar{t}$ events to investigate the $C P$ violation in top quark decay. For simplicity, we only consider two heavy Majorana neutrinos, while the general situation with more heavy Majorana neutrinos can be analyzed in a similar way.

This paper is organized as follows. A phenomenological heavy Majorana model is briefly introduced in Sec. II. The decay of top quark via two different Majorana neutrinos is discussed in Sec. III. Section IV is devoted to the numerical results and discussions at the LHC. Finally, a short summary is given.

\section{HEAVY MAJORANA NEUTRINO MODEL}

Following the same notation in Ref. [32], with two righthanded Majorana neutrinos, the mixing relations between the neutrino flavor eigenstates and mass eigenstates can be given by

$$
\nu_{\ell L}=\sum_{m=1}^{3} V_{\ell m} \nu_{m L}+\sum_{m^{\prime}=1}^{2} R_{\ell m^{\prime}} N_{m^{\prime} L}^{c},
$$

where $\ell=e, \mu, \tau$ and $V V^{\dagger}+R R^{\dagger}=I$. In terms of the mass eigenstates, the weak charged-current interaction Lagrangian can be written as

$$
\begin{aligned}
-\mathcal{L}_{\mathrm{cc}}= & \frac{g}{\sqrt{2}} W_{\mu}^{+} \sum_{\ell=e}^{\tau} \sum_{m=1}^{3} V_{\ell m}^{*} \overline{\nu_{m}} \gamma^{\mu} P_{L} \ell \\
& +\frac{g}{\sqrt{2}} W_{\mu}^{+} \sum_{\ell=e}^{\tau} \sum_{m^{\prime}=1}^{2} R_{\ell m^{\prime}}^{*} \overline{N_{m^{\prime}}^{c}} \gamma^{\mu} P_{L} \ell+\text { H.c.. }
\end{aligned}
$$

Here, $V_{\ell m}$ is the light neutrino mixing matrix that can be measured from the oscillation experiments. As mentioned above, the contributions of the light Majorana neutrinos to the lepton-number-violating processes can be neglected due to their small masses. Note that $R_{\ell m^{\prime}}$ indicates the mixing between charged leptons and heavy Majorana neutrinos and can be parametrized as [51]
$R_{\ell m^{\prime}}=\left|R_{\ell m^{\prime}}\right| e^{i \phi_{\ell m^{\prime}}}, \quad \ell=e, \mu, \tau, \quad m^{\prime}=1,2$.

The complex phase $\phi_{\ell m^{\prime}}$ can serve as one of the new sources of $C P$ violation, which can be determined from possible collider experiments.

The masses of the heavy Majorana neutrinos $m_{N}$ and the mixing elements $R_{\ell m^{\prime}}$ are strongly restricted by experimental observations (for review, we refer to Ref. [52]). So far, the most stringent bound on mixing with electrons can be derived from $0 \nu \beta \beta$-decay experiments [53],

$$
\sum_{i} \frac{\left|R_{e i}\right|^{2}}{m_{N_{i}}}<5 \times 10^{-5} \mathrm{TeV}^{-1} .
$$

It is worth mentioning that the $0 \nu \beta \beta$ constraint is usually model dependent and may be significantly weakened in certain cases [54]. For $m_{N}<m_{Z}$, a 95\% C.L. on the mixing parameters $R_{\ell m^{\prime}}$ can be obtained from a reanalysis of the Large Electron-Positron Collider data [55],

$$
\left|R_{e 1}\right|^{2}, \quad\left|R_{\mu 1}\right|^{2}, \quad\left|R_{\tau 1}\right|^{2}<\mathcal{O}\left(10^{-5}\right) .
$$

A global fit to lepton flavor and electroweak precision data has been performed to constrain the size of $R_{\ell m^{\prime}}$ for heavy neutrino mass above the electroweak scale [56]. At 95\% C.L., the limits are

$$
\begin{gathered}
\sum_{i}\left|R_{e i}\right|^{2}<2.5 \times 10^{-3}, \quad \sum_{i}\left|R_{\mu i}\right|^{2}<4.4 \times 10^{-4}, \\
\sum_{i}\left|R_{\tau i}\right|^{2}<5.6 \times 10^{-3} .
\end{gathered}
$$

At the LHC experiments, the most restrictive direct limits on the mixing parameters $\left|R_{e 1}\right|^{2}$ and $\left|R_{\mu 1}\right|^{2}$ for heavy Majorana neutrino masses between $20 \mathrm{GeV}$ and $1600 \mathrm{GeV}$ are varying from $2.3 \times 10^{-5}$ to unity [57]. In this paper, we will adopt a phenomenological approach where the masses and mixing parameters of the heavy neutrinos are simply parametrized as free parameters. To be conservative, the values of the mixing parameters are set as

$$
\begin{aligned}
& \left|R_{e i}\right|^{2}=1.0 \times 10^{-7} \\
& \left|R_{\mu i}\right|^{2}=\left|R_{\tau i}\right|^{2}=1.0 \times 10^{-5}, \quad \text { for } i=1,2
\end{aligned}
$$

\section{CP VIOLATION IN TOP (ANTITOP) QUARK DECAY}

Given the charged-current interaction Lagrangian in Eq. (2), the decay of top and antitop quark with $\Delta L=2$ via heavy Majorana neutrinos can be, respectively, expressed by 


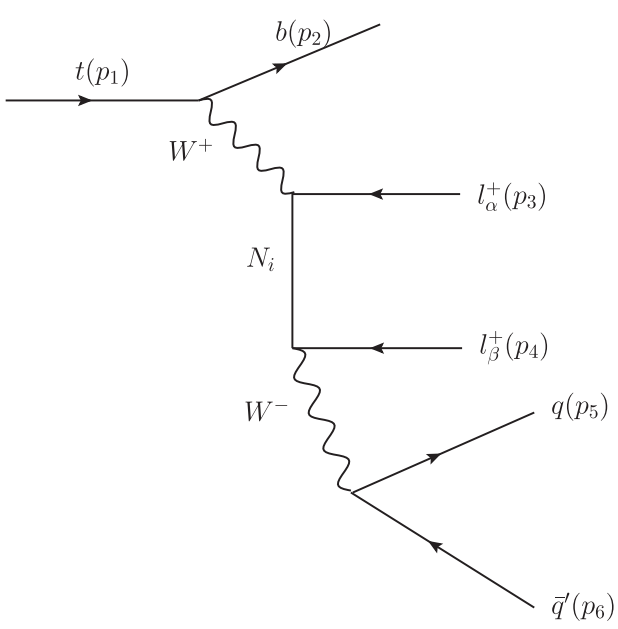

(a)

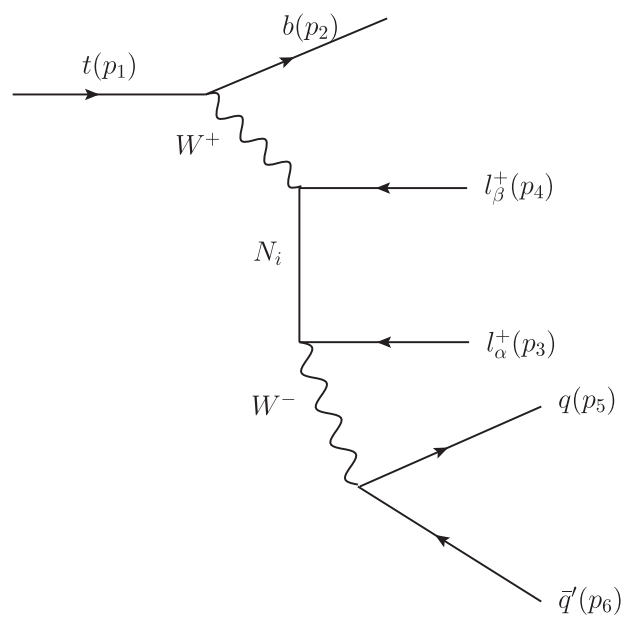

(b)

FIG. 1. Feynman diagrams for top quark rare decay via two heavy Majorana neutrinos $N_{1}$ and $N_{2}$.

$$
\begin{aligned}
t\left(p_{1}\right) & \rightarrow b\left(p_{2}\right)+l_{\alpha}^{+}\left(p_{3}\right)+N_{i}\left(p_{N}\right) \\
& \rightarrow b\left(p_{2}\right)+l_{\alpha}^{+}\left(p_{3}\right)+l_{\beta}^{+}\left(p_{4}\right)+q\left(p_{5}\right)+\bar{q}^{\prime}\left(p_{6}\right), \\
\bar{t}\left(p_{1}\right) & \rightarrow \bar{b}\left(p_{2}\right)+l_{\alpha}^{-}\left(p_{3}\right)+N_{i}\left(p_{N}\right) \\
& \rightarrow \bar{b}\left(p_{2}\right)+l_{\alpha}^{-}\left(p_{3}\right)+l_{\beta}^{-}\left(p_{4}\right)+\bar{q}\left(p_{5}\right)+q^{\prime}\left(p_{6}\right),
\end{aligned}
$$

where $\alpha, \beta=e, \mu, \tau$, and $i=1$, 2. $p_{1}, p_{2}$ etc., represent the four-momentum of the corresponding particles. The corresponding Feynman diagrams for the top quark rare decay are shown in Fig. 1, where Fig. 1(b) is a crossed channel of Fig. 1(a) by $l_{\alpha} \leftrightarrow l_{\beta}$. The differential decay width for the process in Eq. (8) can be given by

$$
d \Gamma\left(t \rightarrow b l_{\alpha} l_{\beta} j_{1} j_{2}\right)=\frac{1}{2 m_{t}} \overline{|\mathcal{M}|^{2}} d \mathcal{L} \text { ips }_{5} .
$$

Here, $d \mathcal{L i p s}_{5}$ represents the five-body Lorentz invariant phase space of the final particles. Note that $\overline{|\mathcal{M}|^{2}}$ is the squared scattering amplitude averaged (summed) over the initial (final) particles and can be expressed as

$$
\begin{aligned}
\overline{\left|\mathcal{M}_{\ell_{\alpha}^{ \pm} \ell_{\beta}^{ \pm}}\right|^{2}}= & \frac{g^{8}}{m_{W}^{4}}\left|V_{t b}\right|^{2}\left|V_{q q^{\prime}}\right|^{2}\left(1-\frac{1}{2} \delta_{\alpha \beta}\right) \\
& \times\left|D_{W}\left(p_{w}^{2}\right)\right|^{2}\left|D_{W}\left(p_{w}^{\prime}{ }^{2}\right)\right|^{2} \\
& \times\left\{m_{N_{1}}^{2}\left|R_{\alpha 1} R_{\beta 1}\right|^{2} \mathcal{T}_{1}+m_{N_{2}}^{2}\left|R_{\alpha 2} R_{\beta 2}\right|^{2} \mathcal{T}_{2}\right. \\
& \left.+m_{N_{1}} m_{N_{2}}\left|R_{\alpha 1} R_{\alpha 2} R_{\beta 1} R_{\beta 2}\right| \operatorname{Re}\left[e^{ \pm i \Delta \phi} \mathcal{T}_{12}\right]\right\},
\end{aligned}
$$

where $p_{w}=p_{1}-p_{2}, \quad p_{w}^{\prime}=p_{5}+p_{6}$. Note that $D_{W}\left(p^{2}\right)=1 /\left(p^{2}-m_{W}^{2}+i m_{W} \Gamma_{W}\right)$, where $m_{W}, \Gamma_{W}$ are the mass and total decay width of $W$ boson. Note that $V_{t b}\left(V_{q q^{\prime}}\right)$ is the Cabibbo-Kobayashi-Maskawa (CKM) matrix element, and $R_{\alpha i}(\alpha=e, \mu, \tau$ and $i=1,2)$ is the mixing matrix element defined in Eq. (3). Also note that
$\Delta \phi=\phi_{\alpha 2}-\phi_{\alpha 1}+\phi_{\beta 2}-\phi_{\beta 1}$ is the $C P$ phase difference induced by the significant interference between $N_{1}$ and $N_{2}$. The explicit expressions of $\mathcal{T}_{i}(i=1,2)$ and $\mathcal{T}_{12}$ are shown in Appendix.

With only one heavy Majorana neutrino, the process in Eq. (8) has been well studied in the literature [31,39]. However, as can be seen in Eq. (9) and Eq. (10), the squared scattering amplitude $\overline{|\mathcal{M}|^{2}}$, and thus the differential decay width $d \Gamma$, of top (antitop) quark decay is irrelevant to the complex phase $\Delta \phi$, which means that there is no $C P$ violation in this case. Therefore, in order to generate the $C P$-violating asymmetry, there must exist at least two different heavy Majorana neutrinos, and the $C P$ phase difference $\Delta \phi$ indicated in Eq. (10) cannot be zero. In the subsequent discussions, we generalize the previous works and consider the case of two intermediate on-shell Majorana neutrinos $N_{1}$ and $N_{2}$. The significant interference between the $N_{1}$ and $N_{2}$ contributions can lead to a difference in the rates of top quark decay and its $C P$-conjugate process, which is a signal of $C P$ violation. Furthermore, the $C P$ violation effect can be appreciable when the two neutrino masses are nearly degenerate.

As demonstrated in Ref. [31], in the narrow-width approximation, the total decay width of top quark can be factorized as

$$
\begin{aligned}
\Gamma\left(t \rightarrow b l_{\alpha} l_{\beta} j_{1} j_{2}\right) & \approx \Gamma\left(t \rightarrow b l_{\alpha} N\right) \\
\operatorname{Br}\left(N \rightarrow l_{\beta} j_{1} j_{2}\right) & =\left(2-\delta_{\alpha \beta}\right) S_{\alpha \beta} \Gamma_{0} .
\end{aligned}
$$

$\Gamma_{0}$ is the reduced decay width and basically independent of all the mixing parameters. Note that $S_{\alpha \beta}$ is the so-called "effective mixing parameter" and is defined as

$$
S_{\alpha \beta}=\frac{\left|R_{\alpha N} R_{\beta N}\right|^{2}}{\sum_{\ell=e}^{\tau}\left|R_{\ell N}\right|^{2}} .
$$




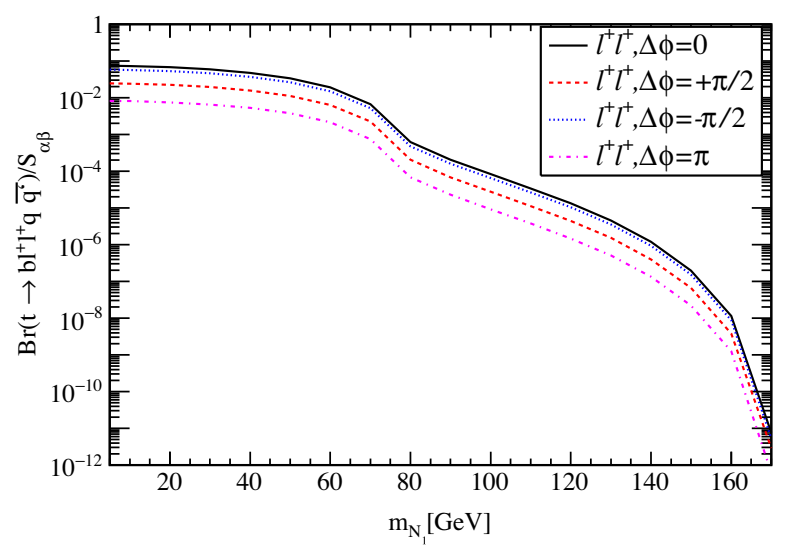

(a)

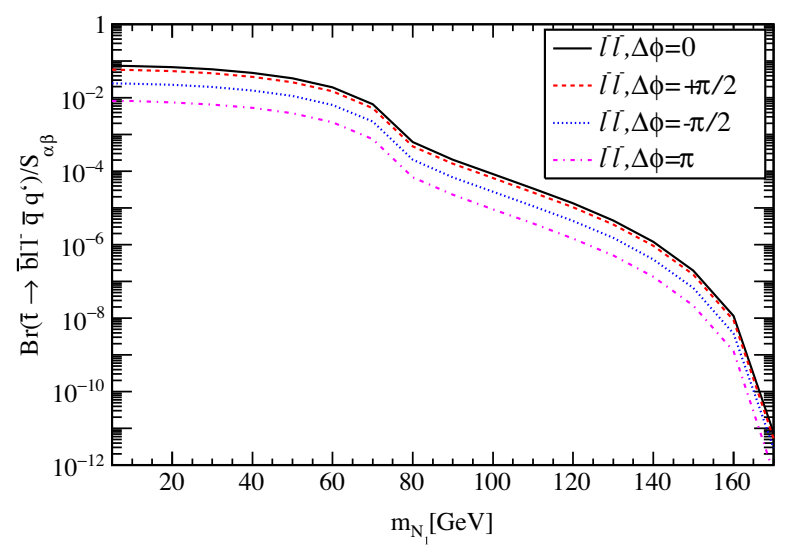

(b)

FIG. 2. Normalized branching ratio of (a) top quark decay $t \rightarrow b l_{\alpha}^{+} l_{\beta}^{+} q \bar{q}^{\prime}$ and (b) antitop quark decay $\bar{t} \rightarrow \bar{b} l_{\alpha}^{-} l_{\beta}^{-} \bar{q} q^{\prime}$ via $N_{1}$ and $N_{2}$ for $\Delta \phi=0,+\pi / 2,-\pi / 2, \pi$.

In our calculations, we employ the same definition for $S_{\alpha \beta}$ in the case with $N_{1}$ and $N_{2}$. For the $t \rightarrow b l^{+} l^{+} q \bar{q}^{\prime}$ process, we choose massless light quark pair $q \bar{q}^{\prime}=u \bar{d}, c \bar{s}$. For simplicity, the CKM matrix is considered as diagonal with unit entries, and only the top quark mass is taken into account. Moreover, the following assumptions are applied:

$$
m_{N_{2}}=m_{N_{1}}+\Gamma_{N_{1}} / 2, \quad \Gamma_{N_{2}} \approx \Gamma_{N_{1}} .
$$

We defined the branching ratio of top quark decay $t \rightarrow$ $b l_{\alpha}^{+} l_{\beta}^{+} q \bar{q}^{\prime}$ and antitop quark decay $\bar{t} \rightarrow \bar{b} l_{\alpha}^{-} l_{\beta}^{-} \bar{q} q^{\prime}$ as follows:

$$
\begin{aligned}
& \operatorname{Br}\left(t \rightarrow b l^{+} l^{+} q \bar{q}^{\prime}\right)=\frac{\Gamma\left(t \rightarrow b l^{+} l^{+} q \bar{q}^{\prime}\right)}{\Gamma_{t}}, \\
& \operatorname{Br}\left(\bar{t} \rightarrow \bar{b} l^{-} l^{-} \bar{q} q^{\prime}\right)=\frac{\Gamma\left(\bar{t} \rightarrow \bar{b} l^{-} l^{-} \bar{q} q^{\prime}\right)}{\Gamma_{t}},
\end{aligned}
$$

where $\Gamma_{t}$ is the total decay width of the top quark. The normalized branching ratios $\operatorname{Br}\left(t \rightarrow b l^{+} l^{+} q \bar{q}^{\prime}\right) / S_{\alpha \beta}$ and

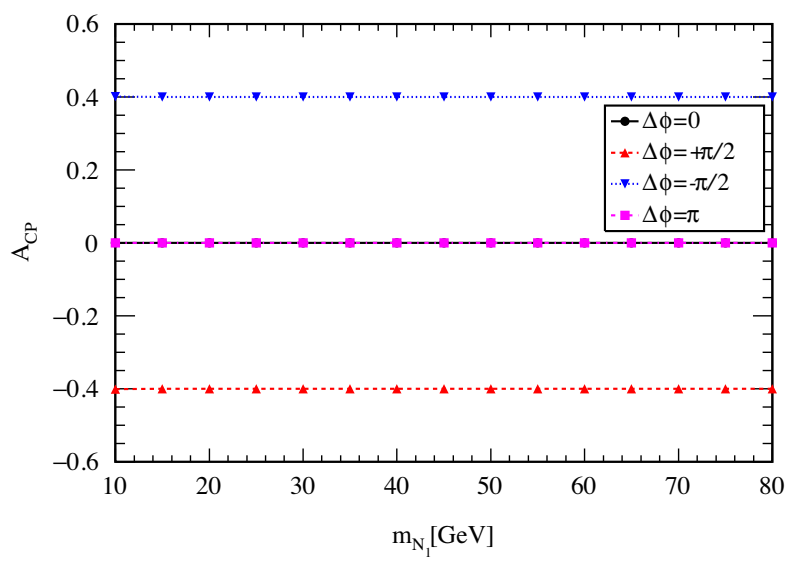

(a)
$\operatorname{Br}\left(\bar{t} \rightarrow \bar{b} l^{-} l^{-} \bar{q} q^{\prime}\right) / S_{\alpha \beta}$ as a function of $m_{N_{1}}$ are shown in Fig. 2, where the $C P$ phase difference is set to $\Delta \phi=0,+\pi / 2,-\pi / 2, \pi$. From this plot, one can find that for fixed $\Delta \phi$, as the Majorana neutrino mass increases, the normalized branching ratio decreases. When $m_{N_{1}}>m_{W}$, the branching ratio becomes less than $10^{-3} S_{\alpha \beta}$ for $\Delta \phi=0$ and $10^{-4} S_{\alpha \beta}$ for $\Delta \phi=\pi$. At the low Majorana neutrino mass region, the search for Majorana neutrinos has been studied extensively in heavy meson decays. In order to effectively measure the $C P$ violation in top quark decay, the Majorana neutrino mass range of our interest is limited to $10 \mathrm{GeV}<m_{N}<80 \mathrm{GeV}$. Moreover, the branching ratio for $\Delta \phi=+\pi / 2(-\pi / 2)[\pi]$ is just about $60 \%$ (30\%) [10\%] of that for $\Delta \phi=0$ in the case of top quark decay, but $30 \%$ $(60 \%)[10 \%]$ in the antitop quark decay case.

The difference between the rates of $t \rightarrow b l_{\alpha}^{+} l_{\beta}^{+} q \bar{q}^{\prime}$ and its $C P$-conjugate process $\bar{t} \rightarrow \bar{b} l_{\alpha}^{-} l_{\beta}^{-} \bar{q} q^{\prime}$ can then induce the $C P$ asymmetry $\mathcal{A}_{\mathrm{CP}}$, which can be defined as

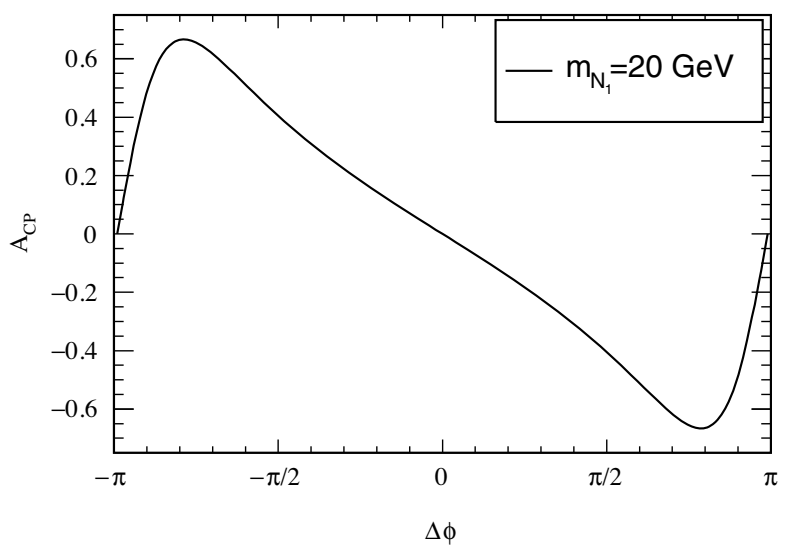

(b)

FIG. 3. (a) The value of $\mathcal{A}_{\mathrm{CP}}$ as a function of $m_{N_{1}}$ for $\Delta \phi=0,+\pi / 2,-\pi / 2, \pi$. (b) The value of $\mathcal{A}_{\mathrm{CP}}$ as a function of $\Delta \phi$ for $m_{N_{1}}=20 \mathrm{GeV}$. 


$$
\mathcal{A}_{\mathrm{CP}}=\frac{\Gamma\left(t \rightarrow b l_{\alpha}^{+} l_{\beta}^{+} q \bar{q}^{\prime}\right)-\Gamma\left(\bar{t} \rightarrow \bar{b} l_{\alpha}^{-} l_{\beta}^{-} \bar{q} q^{\prime}\right)}{\Gamma\left(t \rightarrow b l_{\alpha}^{+} l_{\beta}^{+} q \bar{q}^{\prime}\right)+\Gamma\left(\bar{t} \rightarrow \bar{b} l_{\alpha}^{-} l_{\beta}^{-} \bar{q} q^{\prime}\right)} .
$$

The numerical results of $\mathcal{A}_{\mathrm{CP}}$ as a function of $m_{N_{1}}$ for various values of $\Delta \phi$ are shown in Fig. 3(a). For comparison, we also display the value of $\mathcal{A}_{\mathrm{CP}}$ as a function of $\Delta \phi$ for $m_{N_{1}}=20 \mathrm{GeV}$ in Fig. 3(b). It is found that, for $\Delta \phi=0, \pi$, the $C P$ asymmetry disappears. Furthermore, we see that the size of $\mathcal{A}_{\mathrm{CP}}$ is basically independent of the Majorana neutrino mass, and $\mathcal{A}_{\mathrm{CP}} \rightarrow-\mathcal{A}_{\mathrm{CP}}$ for negative values of $\Delta \phi$. The maximal values of $\mathcal{A}_{\mathrm{CP}} \approx 0.6$ can be reached for $\Delta \phi \approx \pm 4 \pi / 5$.

\section{CP VIOLATION IN TOP ANTITOP PAIR PRODUCTION AND RARE DECAY AT THE LHC}

As a top rich environment, the LHC will offer a great opportunity to precisely explore the prospects for measuring such a $C P$ asymmetry. At the LHC, the $t \bar{t}$ pairs are

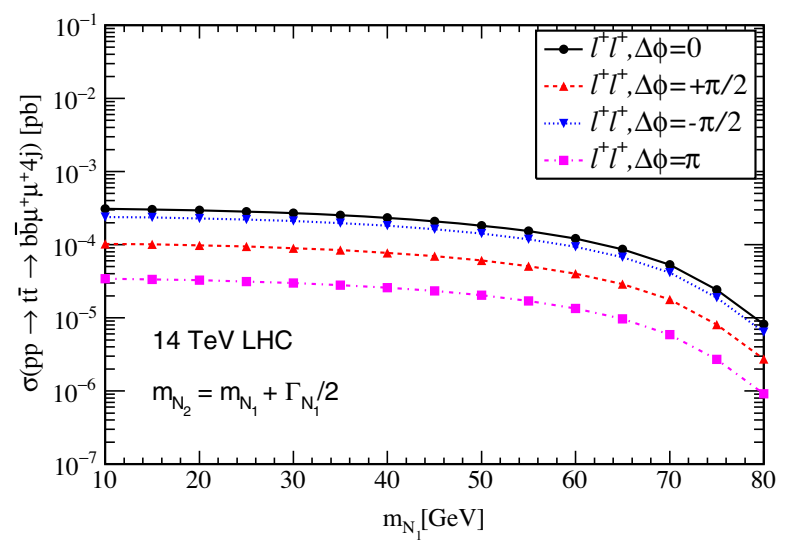

(a)

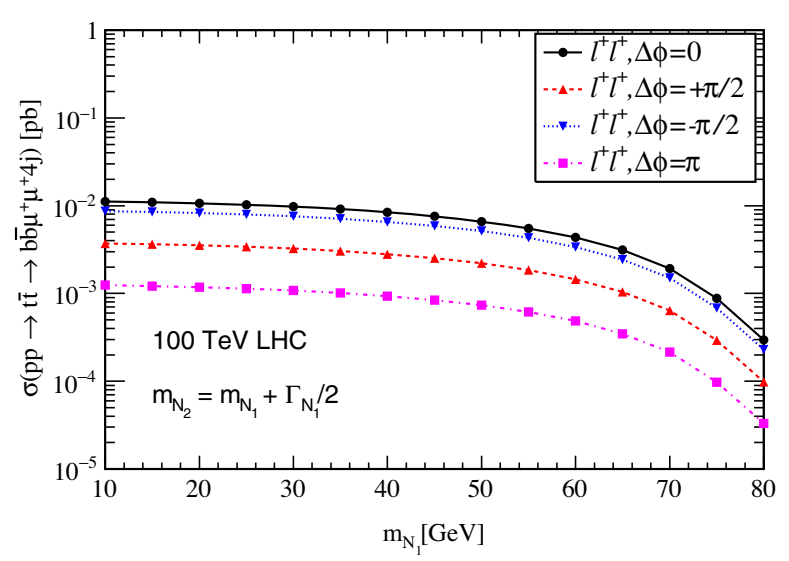

(c) dominantly produced by the strong interactions [58-60]. In this paper, we consider the following process:

$$
p p \rightarrow t \bar{t} \rightarrow b \bar{b}+l^{ \pm} l^{ \pm}+4 j,
$$

where one top quark produces leptons, and the other one is required to decay hadronically. Since the detection of muon leptons is most efficient at the LHC, we will thus concentrate on the clean dimuon production channel. In Fig. 4, we display, respectively, the total cross sections for the samesign dilepton production process as a function of $m_{N_{1}}$ at $14 \mathrm{TeV}$ and $100 \mathrm{TeV}$ LHC, where the $C P$ phase difference is set to $\Delta \phi=0,+\pi / 2,-\pi / 2, \pi$. Here, we employ the CTEQ6L1 for the parton distribution functions in the proton [61].

To investigate the $C P$ violation at the $\mathrm{LHC}$, the $C P$ asymmetry can be defined as

$$
\overline{\mathcal{A}_{\mathrm{CP}}}=\frac{\sigma\left(p p \rightarrow b \bar{b} \mu^{+} \mu^{+} 4 j\right)-\sigma\left(p p \rightarrow b \bar{b} \mu^{-} \mu^{-} 4 j\right)}{\sigma\left(p p \rightarrow b \bar{b} \mu^{+} \mu^{+} 4 j\right)+\sigma\left(p p \rightarrow b \bar{b} \mu^{-} \mu^{-} 4 j\right)} .
$$

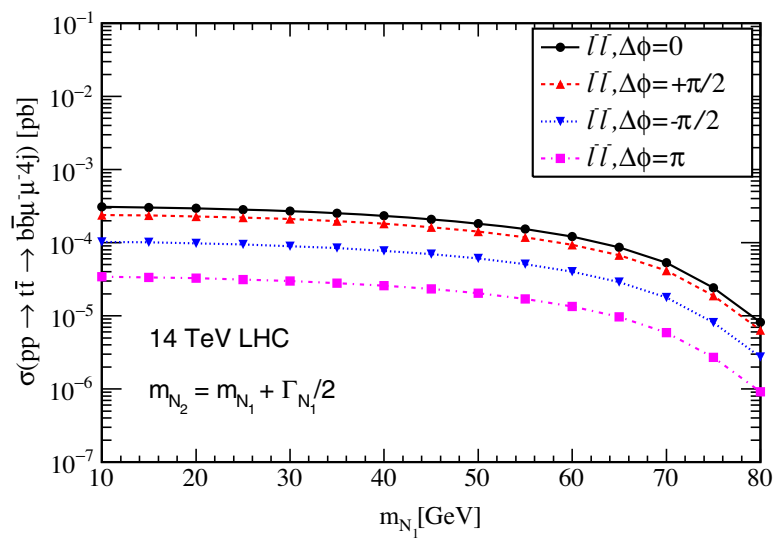

(b)

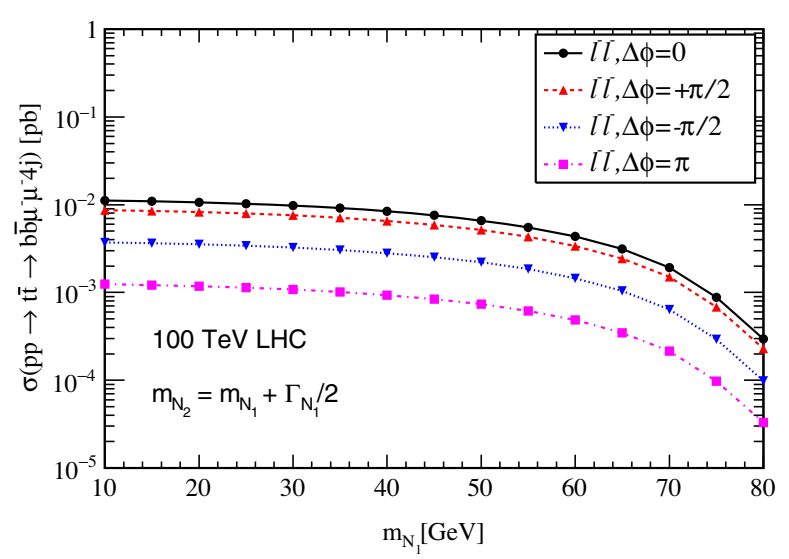

(d)

FIG. 4. The total cross sections for (a) $p p \rightarrow t \bar{t} \rightarrow b \bar{b} \mu^{+} \mu^{+} 4 j$ and (b) $p p \rightarrow t \bar{t} \rightarrow b \bar{b} \mu^{-} \mu^{-} 4 j$ at 14 TeV LHC, (c) $p p \rightarrow t \bar{t} \rightarrow b \bar{b} \mu^{+} \mu^{+} 4 j$ and (d) $p p \rightarrow t \bar{t} \rightarrow b \bar{b} \mu^{-} \mu^{-} 4 j$ at $100 \mathrm{TeV}$ LHC, as a function of $m_{N_{1}}$. Here the $C P$ phase difference is set to $\Delta \phi=0,+\pi / 2,-\pi / 2, \pi$. 


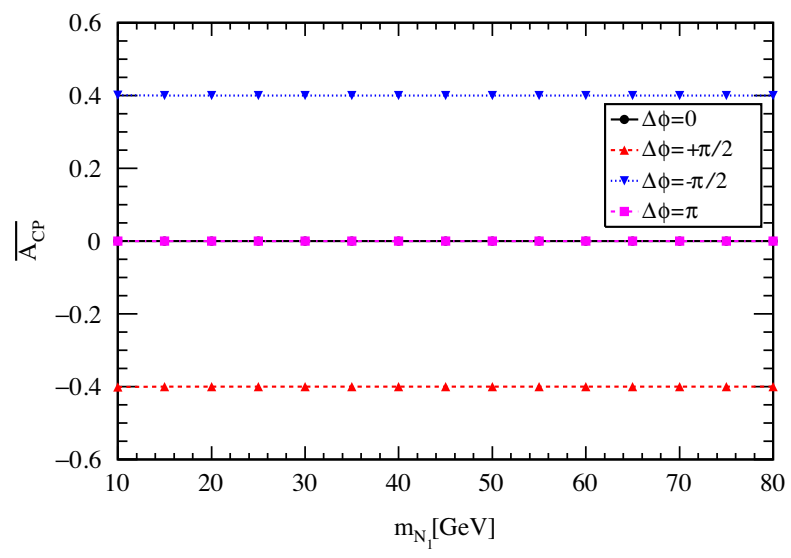

(a)

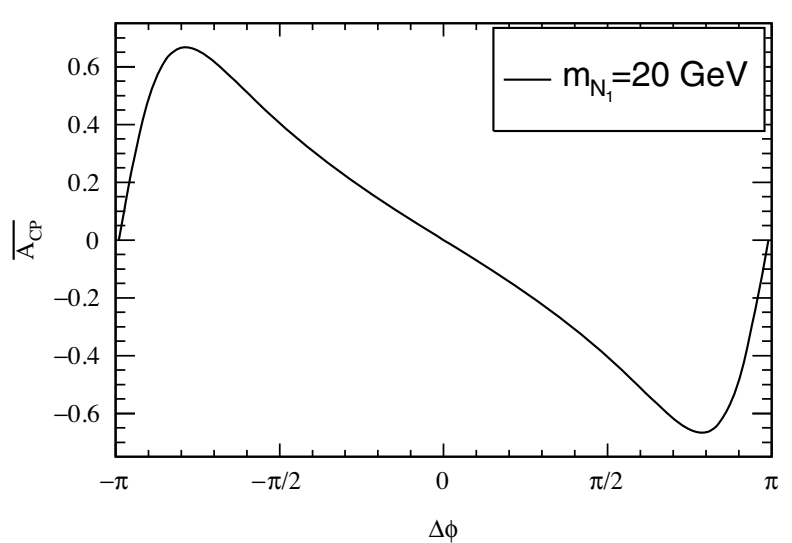

(b)

FIG. 5. (a) The value of $\mathcal{A}_{\mathrm{CP}}$ as a function of $m_{N_{1}}$ for $\Delta \phi=0,+\pi / 2,-\pi / 2, \pi$. (b) The value of $\mathcal{A}_{\mathrm{CP}}$ as a function of $\Delta \phi$ for $m_{N_{1}}=20 \mathrm{GeV}$.

The numerical results of $\overline{\mathcal{A}_{\mathrm{CP}}}$ are shown in Fig. 5. One finds that the tendency of the $C P$ asymmetry $\overline{\mathcal{A}_{\mathrm{CP}}}$ in Fig. 5 is the same as that in Fig. 3. This is exactly what we expected. The reason is that the $C P$ asymmetry only stems from the rate difference between top quark decay and its $C P$-conjugate process.

Our signal consists of two same-sign dilepton plus six jets (including two $\mathrm{b}$ jets) associated with no significant missing transverse energy. As the jets originating from the top quark five-body decay $t \rightarrow b l^{+} l^{+} j j$ are much softer than those in the decay of $\bar{t} \rightarrow \bar{b} j j$, the six jets in final state may be merged into four jets plus one fat jet. In our calculations, the number of the final state jets is limited to $n_{j}=5$. Though there is no source of missing transverse momentum in our signal process, the missing transverse momentum may appear due to the detector-level mismeasurements. To simulate the detector effects, we smear the lepton and jet energies according to the assumption of the Gaussian resolution parametrization,

$$
\frac{\delta(E)}{E}=\frac{a}{\sqrt{E}} \oplus b
$$

where $a=5 \%, b=0.55 \%$ for leptons, and $a=100 \%$, $b=5 \%$ for jets, respectively $[62,63]$. In order to identify the isolated lepton or jet, the angular separation between particle $i$ and particle $j$ can be defined as

$$
\Delta R_{i j}=\sqrt{\Delta \phi_{i j}^{2}+\Delta \eta_{i j}^{2}} .
$$

In the following numerical calculations, we apply the basic acceptance cuts (referred to as cut I):

$$
\begin{aligned}
p_{T}^{\ell} & >10 \mathrm{GeV}, \quad\left|\eta^{\ell}\right|<2.8, \quad p_{T}^{j}>15 \mathrm{GeV}, \\
\left|\eta^{j}\right| & <3.0,0.4<\Delta R_{\ell j}<2.5, \quad n_{j}=5 .
\end{aligned}
$$

To maximize the contributions to our signal rate, we further demand the missing transverse energy satisfies (referred to as cut II)

$$
\mathbb{E}_{T}<20 \mathrm{GeV} \text {. }
$$

The dominant backgrounds in the standard model for our signal process are $p p \rightarrow t \bar{t} W^{ \pm} j \rightarrow b \bar{b} \mu^{ \pm} \mu^{ \pm} j j j+\mathbb{E}_{T}$ and $p p \rightarrow b \bar{b} W^{ \pm} W^{ \pm} j j j \rightarrow b \bar{b} \mu^{ \pm} \mu^{ \pm} j j j+\mathscr{E}_{T}$, which are simulated by MADGRAPH5_aMC@NLO [64]. After analyzing these kinds of backgrounds, we find that the backgrounds $b \bar{b} W^{ \pm} W^{ \pm} j j j$ are much smaller and can be neglected. The parton shower and hadronization are performed with PYTHIA-8.2 [65] and the fast detector simulation are simulated with PGS [66]. Jets clustering is done by FastJet [67] with the anti- $k_{t}$ algorithm [68].

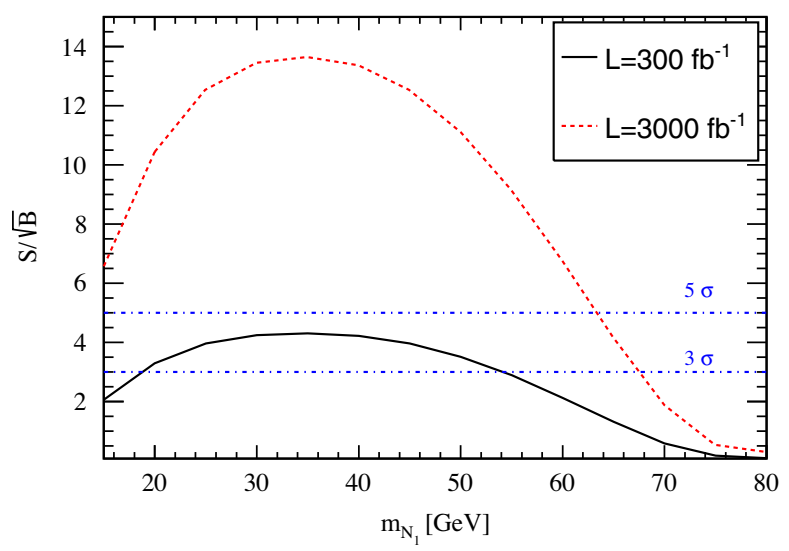

FIG. 6. The statistical significance $S / \sqrt{B}$ as a function of $m_{N_{1}}$ with the integrated luminosity of $\mathcal{L}=300 \mathrm{fb}^{-1}$ and $\mathcal{L}=$ $3000 \mathrm{fb}^{-1}$ at $14 \mathrm{TeV} \mathrm{LHC}$, where the $C P$ phase difference is set to $\Delta \phi=\pi / 2$. 
TABLE I. The cross sections for the signal and background processes at $14 \mathrm{TeV}$ and $100 \mathrm{TeV}$ LHC after all cuts. Also shown is the statistical significance $S / \sqrt{B}$ with integrated luminosity of $\mathcal{L}=300 \mathrm{fb}^{-1}$ and $\mathcal{L}=3000 \mathrm{fb}^{-1}$. For illustration, we have used $m_{N_{1}}=$ $30 \mathrm{GeV}$ and $\Delta \phi=\pi / 2$.

\begin{tabular}{|c|c|c|c|c|}
\hline & \multicolumn{2}{|c|}{$14 \mathrm{TeV}$} & \multicolumn{2}{|c|}{$100 \mathrm{TeV}$} \\
\hline & $\sigma\left(p p \rightarrow b \bar{b} l^{ \pm} l^{ \pm} 4 j\right)[\mathrm{fb}]$ & $\sigma\left(p p \rightarrow \bar{t} \bar{t} W^{ \pm} j\right)[\mathrm{fb}]$ & $\sigma\left(p p \rightarrow b \bar{b} l^{ \pm} l^{ \pm} 4 j\right)[\mathrm{fb}]$ & $\sigma\left(p p \rightarrow t \bar{t} W^{ \pm} j\right)[\mathrm{fb}]$ \\
\hline Cut I & $1.21 \times 10^{-2}$ & $1.81 \times 10^{-1}$ & $3.22 \times 10^{-1}$ & 3.01 \\
\hline Cut II & $7.54 \times 10^{-3}$ & $8.48 \times 10^{-3}$ & $1.98 \times 10^{-1}$ & $2.99 \times 10^{-2}$ \\
\hline Cut III & $4.35 \times 10^{-3}$ & $3.16 \times 10^{-4}$ & $1.15 \times 10^{-1}$ & $2.82 \times 10^{-3}$ \\
\hline$S / \sqrt{B}$ with $\mathcal{L}=300 \mathrm{fb}^{-1}$ & \multicolumn{2}{|c|}{4.24} & \multicolumn{2}{|c|}{37.18} \\
\hline$S / \sqrt{B}$ with $\mathcal{L}=3000 \mathrm{fb}^{-1}$ & \multicolumn{2}{|c|}{13.40} & \multicolumn{2}{|c|}{117.60} \\
\hline
\end{tabular}

To purify the signal, we employ the procedure described in Ref. [31] to fully reconstruct the two tops. First, the three jets with invariant mass closest to $m_{t}$ are selected to reconstruct the hadronic top. Second, all the remaining ingredients are grouped to reconstruct the leptonic top. The following cut on the top reconstruction is applied (referred to as cut III):

$$
\left|M_{\text {inv }}-m_{t}\right|<20 \mathrm{GeV} \text {. }
$$

Taking $\Delta \phi=\pi / 2$ as an example, with the integrated luminosity of $\mathcal{L}=300 \mathrm{fb}^{-1}$ and $\mathcal{L}=3000 \mathrm{fb}^{-1}$, the statistical significance $S / \sqrt{B}$ as a function of $m_{N_{1}}$ at $14 \mathrm{TeV}$ LHC is displayed in Fig. 6, where $S$ and $B$ denote the signal and background event numbers after all the cuts in Eqs. (20)-(22), respectively. We find that a $3 \sigma$ discovery can be made for $19 \mathrm{GeV}<m_{N_{1}}<54 \mathrm{GeV}$ with $\mathcal{L}=$ $300 \mathrm{fb}^{-1}$ at $14 \mathrm{TeV}$ LHC. With $\mathcal{L}=3000 \mathrm{fb}^{-1}$, the heavy Majorana neutrino mass can reach $m_{N_{1}} \simeq 64 \mathrm{GeV}$ for $5 \sigma$ discovery. Moreover, at $m_{N_{1}} \simeq 35 \mathrm{GeV}$, the statistical significance takes the maximum value $S / \sqrt{B} \simeq 13.5$. The cross sections for the signal and background processes at $14 \mathrm{TeV}$ and $100 \mathrm{TeV}$ LHC after all cuts are shown in Table I. Also shown is the statistical significance $S / \sqrt{B}$ with integrated luminosity of $\mathcal{L}=300 \mathrm{fb}^{-1}$ and $\mathcal{L}=3000 \mathrm{fb}^{-1}$. For illustration, we have used $m_{N_{1}}=$ $30 \mathrm{GeV}$ and $\Delta \phi=\pi / 2$. It is shown that after all the cuts,

TABLE II. The value of $\overline{\mathcal{A}_{\mathrm{CP}}}$ at $14 \mathrm{TeV}$ and $100 \mathrm{TeV}$ LHC after all cuts for $m_{N_{1}}=30 \mathrm{GeV}$ and $\Delta \phi= \pm \pi / 4, \pm \pi / 2, \pm 4 \pi / 5$.

\begin{tabular}{lrr}
\hline \hline & $14 \mathrm{TeV}$ & $100 \mathrm{TeV}$ \\
\hline$\Delta \phi=-4 \pi / 5$ & 0.67 & 0.67 \\
$\Delta \phi=-\pi / 2$ & 0.40 & 0.40 \\
$\Delta \phi=-\pi / 4$ & 0.18 & 0.18 \\
$\Delta \phi=\pi / 4$ & -0.18 & -0.18 \\
$\Delta \phi=\pi / 2$ & -0.40 & -0.40 \\
$\Delta \phi=4 \pi / 5$ & -0.67 & -0.67 \\
\hline \hline
\end{tabular}

the signal cross section remains only $4.35 \times 10^{-3} \mathrm{fb}^{-1}$ at $14 \mathrm{TeV}$ LHC, which is hard to be detected. However, it is possible to do the study at $100 \mathrm{TeV}$ LHC, and the corresponding statistical significance can reach 37.18 (117.60) with $\mathcal{L}=300 \mathrm{fb}^{-1} \quad\left(\mathcal{L}=3000 \mathrm{fb}^{-1}\right)$. Finally, we show the value of $\overline{\mathcal{A}_{\mathrm{CP}}}$ at $14 \mathrm{TeV}$ and $100 \mathrm{TeV} \mathrm{LHC}$ after all cuts in Table II, where $m_{N_{1}}=30 \mathrm{GeV}$, and $\Delta \phi=$ $\pm \pi / 4, \pm \pi / 2, \pm 4 \pi / 5$ are used for illustration. The $C P$ violation effect can be used as the evidence for new physics beyond the standard model.

\section{SUMMARY}

The existence of heavy Majorana neutrinos provides one of the most promising explanations for the origin of neutrino masses. In this paper, we investigate the $C P$ violation in top quark pair production and their rare lepton-number-violating decay at the LHC. The significant interference of contributions from two nearly-degenerate Majorana neutrinos can lead to a $C P$-violating effect between $t \rightarrow b l^{+} l^{+} X$ and $\bar{t} \rightarrow \bar{b} l^{-} l^{-} X$ processes. It is found that in the Majorana neutrino mass range of $10 \mathrm{GeV}<m_{N}<80 \mathrm{GeV}$, the $C P$ asymmetry is independent of the Majorana neutrino mass at the LHC. Taking $m_{N_{1}}=30 \mathrm{GeV}$ and $\Delta \phi=\pi / 2$ as an example, we explore the discovery prospects of $C P$ violation at $14 \mathrm{TeV}$ and $100 \mathrm{TeV}$ LHC. We find that the signal events are hard to be detected at $14 \mathrm{TeV}$ LHC, while it is possible at $100 \mathrm{TeV}$ LHC. Furthermore, we investigate the $C P$ asymmetry $\overline{\mathcal{A}_{\mathrm{CP}}}$ for $\Delta \phi= \pm \pi / 4, \pm \pi / 2$ and $\pm 4 \pi / 5$ after the accepted cuts for $m_{N_{1}}=30 \mathrm{GeV}$ at the LHC. Once this kind of $C P$ violation effects induced by the Majorana phase in top quark rare decay are observed at the LHC or future high energy colliders, they will be the clear evidence of new physics beyond the standard model.

\section{ACKNOWLEDGMENTS}

P.-C. L., Z.-G. S., Z. W., and X.-H. Y. thank the members of the Institute of theoretical physics of 
Shandong University for their helpful discussions. This work is supported by National Natural Science Foundation of China (Grants No. 11875179 and 11775130 ) and that of Shandong Province (Grant No. ZR2021QA040).

\section{APPENDIX: CALCULATION OF THE SQUARED SCATTERING AMPLITUDE}

In this Appendix, we show explicitly the squared scattering amplitude given in Eq. (10). The functions $\mathcal{T}_{i}$ $(i=1,2)$ and $\mathcal{T}_{12}$ can be, respectively, expressed as

$$
\begin{aligned}
\mathcal{T}_{i}= & \left|D_{N_{i}}\left(p_{N}^{2}\right)\right|^{2} \cdot \mathcal{F}+\left|D_{N_{i}}\left(p_{N}^{\prime}{ }^{2}\right)\right|^{2} \cdot \mathcal{G} \\
& -\operatorname{Re}\left[D_{N_{i}}\left(p_{N}^{2}\right) D_{N_{i}}^{*}\left(p_{N}^{\prime}{ }^{2}\right)\right] \cdot \mathcal{I} \\
& -\operatorname{Im}\left[D_{N_{i}}\left(p_{N}^{2}\right) D_{N_{i}}^{*}\left(p_{N}^{\prime}{ }^{2}\right)\right] \cdot \mathcal{J}
\end{aligned}
$$

$$
\begin{aligned}
\mathcal{T}_{12}= & 2 D_{N_{1}}\left(p_{N}^{2}\right) D_{N_{2}}^{*}\left(p_{N}^{2}\right) \cdot \mathcal{F}+2 D_{N_{1}}\left(p_{N}^{\prime}{ }^{2}\right) D_{N_{2}}^{*}\left(p_{N}^{\prime}{ }^{2}\right) \cdot \mathcal{G} \\
& -\left[D_{N_{1}}\left(p_{N}^{2}\right) D_{N_{2}}^{*}\left(p_{N}^{\prime}{ }^{2}\right)+D_{N_{1}}\left(p_{N}^{\prime}{ }^{2}\right) D_{N_{2}}^{*}\left(p_{N}^{2}\right)\right] \cdot \mathcal{I} \\
& +i\left[D_{N_{1}}\left(p_{N}^{2}\right) D_{N_{2}}^{*}\left(p_{N}^{\prime}{ }^{2}\right)-D_{N_{1}}\left(p_{N}^{\prime}{ }^{2}\right) D_{N_{2}}^{*}\left(p_{N}^{2}\right)\right] \cdot \mathcal{J},
\end{aligned}
$$

where $p_{N}=p_{1}-p_{2}-p_{3}$ and $p_{N}^{\prime}=p_{1}-p_{2}-p_{4}$. Note that $D_{N_{i}}\left(p^{2}\right)(i=1,2)$ is the Breit-Wigner propagator and can be defined as

$$
D_{N_{i}}\left(p^{2}\right)=\frac{1}{p^{2}-m_{N_{i}}^{2}+i m_{N_{i}} \Gamma_{N_{i}}},
$$

with $m_{N_{i}}$ and $\Gamma_{N_{i}}$ being the mass and total decay width of the two Majorana neutrinos $N_{1}$ and $N_{2}$.

The explicit expressions of $\mathcal{F}, \mathcal{G}, \mathcal{I}$, and $\mathcal{J}$ introduced in Eq. (A1) and Eq. (A2) can be given by

$$
\begin{aligned}
\mathcal{F}= & \left\{-2 m_{t}^{2} m_{W}^{2}\left(p_{1} \cdot p_{5}\right)\left(p_{2} \cdot p_{3}\right)-2 m_{W}^{2}\left(m_{t}^{2}-2 m_{W}^{2}\right)\left(p_{1} \cdot p_{3}\right)\left(p_{2} \cdot p_{5}\right)+m_{t}^{2}\left[4 m_{W}^{2}\left(p_{2} \cdot p_{3}\right)\left(p_{2} \cdot p_{5}\right)\right.\right. \\
& \left.\left.+\left(p_{1} \cdot p_{2}\right)\left[\left(-m_{t}^{2}+2 m_{W}^{2}+2\left(p_{1} \cdot p_{2}\right)\right)\left(p_{3} \cdot p_{5}\right)+2\left(p_{3} \cdot p_{w}\right)\left(p_{5} \cdot p_{w}\right)\right]\right]\right\}\left(p_{4} \cdot p_{6}\right), \\
\mathcal{G}= & \left\{-2 m_{t}^{2} m_{W}^{2}\left(p_{1} \cdot p_{5}\right)\left(p_{2} \cdot p_{4}\right)-2 m_{W}^{2}\left(m_{t}^{2}-2 m_{W}^{2}\right)\left(p_{1} \cdot p_{4}\right)\left(p_{2} \cdot p_{5}\right)+m_{t}^{2}\left[4 m_{W}^{2}\left(p_{2} \cdot p_{4}\right)\left(p_{2} \cdot p_{5}\right)\right.\right. \\
& \left.\left.+\left(p_{1} \cdot p_{2}\right)\left[\left(-m_{t}^{2}+2 m_{W}^{2}+2\left(p_{1} \cdot p_{2}\right)\right)\left(p_{4} \cdot p_{5}\right)+2\left(p_{4} \cdot p_{w}\right)\left(p_{5} \cdot p_{w}\right)\right]\right]\right\}\left(p_{3} \cdot p_{6}\right), \\
\mathcal{I}= & 2 m_{W}^{2}\left\{\left(m_{t}^{2}-2 m_{W}^{2}\right)\left(p_{2} \cdot p_{5}\right)\left[\left(p_{1} \cdot p_{3}\right)\left(p_{4} \cdot p_{6}\right)+\left(p_{1} \cdot p_{4}\right)\left(p_{3} \cdot p_{6}\right)-\left(p_{1} \cdot p_{6}\right)\left(p_{3} \cdot p_{4}\right)\right]\right. \\
& \left.-m_{t}^{2}\left[\left(p_{2} \cdot p_{5}\right)-\left(p_{5} \cdot p_{w}\right)\right]\left[\left(p_{2} \cdot p_{3}\right)\left(p_{4} \cdot p_{6}\right)+\left(p_{2} \cdot p_{4}\right)\left(p_{3} \cdot p_{6}\right)-\left(p_{2} \cdot p_{6}\right)\left(p_{3} \cdot p_{4}\right)\right]\right\} \\
+ & m_{t}^{2}\left(m_{t}^{2}-2 m_{W}^{2}-2\left(p_{1} \cdot p_{2}\right)\right)\left(p_{1} \cdot p_{2}\right)\left[\left(p_{3} \cdot p_{6}\right)\left(p_{4} \cdot p_{5}\right)+\left(p_{3} \cdot p_{5}\right)\left(p_{4} \cdot p_{6}\right)-\left(p_{3} \cdot p_{4}\right)\left(p_{5} \cdot p_{6}\right)\right] \\
+ & 2 m_{t}^{2}\left(p_{1} \cdot p_{2}\right)\left(p_{5} \cdot p_{w}\right)\left[\left(p_{3} \cdot p_{4}\right)\left(p_{6} \cdot p_{w}\right)-\left(p_{3} \cdot p_{6}\right)\left(p_{4} \cdot p_{w}\right)-\left(p_{4} \cdot p_{6}\right)\left(p_{3} \cdot p_{w}\right)\right], \\
\mathcal{J}= & 2 m_{W}^{2}\left\{2 m_{W}^{2}\left(p_{2} \cdot p_{5}\right)-m_{t}^{2}\left[\left(p_{1} \cdot p_{5}\right)-\left(p_{2} \cdot p_{5}\right)\right]\right\} \epsilon_{p_{1} p_{2} p_{3} p_{4}}+\left\{4 m_{W}^{4}\left(p_{2} \cdot p_{5}\right)-m_{t}^{4}\left(p_{1} \cdot p_{2}\right)\right. \\
+ & \left.2 m_{t}^{2}\left[\left(p_{1} \cdot p_{2}\right)\left(m_{W}^{2}+\left(p_{1} \cdot p_{2}\right)+\left(p_{1} \cdot p_{5}\right)\right)-\left(m_{W}^{2}+\left(p_{1} \cdot p_{2}\right)\right)\left(p_{2} \cdot p_{5}\right)\right]\right\} \epsilon_{p_{1} p_{3} p_{4} p_{5}} \\
& +m_{t}^{2}\left\{m_{t}^{2}\left(p_{1} \cdot p_{2}\right)-2\left[m_{W}^{2}+\left(p_{1} \cdot p_{2}\right)\right]\left[\left(p_{1} \cdot p_{2}\right)+\left(p_{1} \cdot p_{5}\right)\right]+2\left[m_{W}^{2}+\left(p_{1} \cdot p_{2}\right)\right]\left(p_{2} \cdot p_{5}\right)\right\} \epsilon_{p_{2} p_{3} p_{4} p_{5}},
\end{aligned}
$$

where $\epsilon_{p_{1} p_{2} p_{3} p_{4}}=\epsilon_{\mu \nu \rho \sigma} p_{1}^{\mu} p_{2}^{\nu} p_{3}^{\rho} p_{4}^{\sigma}$.

[1] P. A. Zyla et al. (Particle Data Group), Review of particle physics, Prog. Theor. Exp. Phys. (2020), $083 \mathrm{C} 01$.

[2] P. Minkowski, $\mu \rightarrow e \gamma$ at a rate of one out of $10^{9}$ muon decays? Phys. Lett. 67B, 421 (1977).

[3] T. Yanagida, in Proceedings of the Workshop on Unified Theory and the Baryon Number of the Universe, edited by O. Sawada and A. Sugamoto (KEK, Tsukuba, 1979), p. 95 .
[4] M. Gell-Mann, P. Ramond, and R. Slansky, in Supergravity, edited by P. van Nieuwenhuizen and D.Z. Freeman (North-Holland, Amsterdam, 1979), p. 315.

[5] S. L. Glashow, in Quarks and Leptons, edited by M. Levy et al. (Plenum, New York, 1980), p. 707.

[6] R. N. Mohapatra and G. Senjanovic, Neutrino Mass and Spontaneous Parity Nonconservation, Phys. Rev. Lett. 44, 912 (1980). 
[7] R. N. Mohapatra, Mechanism for Understanding Small Neutrino Mass in Superstring Theories, Phys. Rev. Lett. 56, 561 (1986).

[8] R. N. Mohapatra and J. W. F. Valle, Neutrino mass and baryon number nonconservation in superstring models, Phys. Rev. D 34, 1642 (1986).

[9] J. Bernabeu, A. Santamaria, J. Vidal, A. Mendez, and J. W. F. Valle, Lepton flavor nonconservation at highenergies in a superstring inspired standard model, Phys. Lett. B 187, 303 (1987).

[10] M. B. Gavela, T. Hambye, D. Hernandez, and P. Hernandez, Minimal flavour seesaw models, J. High Energy Phys. 09 (2009) 038.

[11] E. K. Akhmedov, M. Lindner, E. Schnapka, and J. W. F. Valle, Left-right symmetry breaking in NJL approach, Phys. Lett. B 368, 270 (1996).

[12] E. K. Akhmedov, M. Lindner, E. Schnapka, and J. W. F. Valle, Dynamical left-right symmetry breaking, Phys. Rev. D 53, 2752 (1996).

[13] M. Fukugita and T. Yanagida, Baryogenesis without grand unification, Phys. Lett. B 174, 45 (1986).

[14] S. Dodelson and L. M. Widrow, Sterile-Neutrinos as Dark Matter, Phys. Rev. Lett. 72, 17 (1994).

[15] X. D. Shi and G. M. Fuller, A New Dark Matter Candidate: Nonthermal Sterile Neutrinos, Phys. Rev. Lett. 82, 2832 (1999).

[16] K. Abazajian, G. M. Fuller, and M. Patel, Sterile neutrino hot, warm, and cold dark matter, Phys. Rev. D 64, 023501 (2001).

[17] T. Asaka, S. Blanchet, and M. Shaposhnikov, The nuMSM, dark matter and neutrino masses, Phys. Lett. B 631, 151 (2005).

[18] W. H. Furry, On transition probabilities in double betadisintegration, Phys. Rev. 56, 1184 (1939).

[19] M. Doi, T. Kotani, and E. Takasugi, Double beta decay and Majorana neutrino, Prog. Theor. Phys. Suppl. 83, 1 (1985).

[20] S. R. Elliott and J. Engel, Double beta decay, J. Phys. G 30, R183 (2004).

[21] J. Abad, J. G. Esteve, and A.F. Pacheco, Neutrinoless double beta decay of the kaon in a relativistic quark model, Phys. Rev. D 30, 1488 (1984).

[22] L. S. Littenberg and R. E. Shrock, Upper Bounds on Lepton Number Violating Meson Decays, Phys. Rev. Lett. 68, 443 (1992).

[23] C. Dib, V. Gribanov, S. Kovalenko, and I. Schmidt, K meson neutrinoless double muon decay as a probe of neutrino masses and mixings, Phys. Lett. B 493, 82 (2000).

[24] A. Ali, A. V. Borisov, and N. B. Zamorin, Majorana neutrinos and same sign dilepton production at LHC and in rare meson decays, Eur. Phys. J. C 21, 123 (2001).

[25] A. Ilakovac, B. A. Kniehl, and A. Pilaftsis, Semileptonic lepton number/flavor violating tau decays in Majorana neutrino models, Phys. Rev. D 52, 3993 (1995).

[26] A. Ilakovac, Probing lepton number/flavor violation in semileptonic $\tau$ decays into two mesons, Phys. Rev. D 54, 5653 (1996).

[27] V. Gribanov, S. Kovalenko, and I. Schmidt, Sterile neutrinos in tau lepton decays, Nucl. Phys. B607, 355 (2001).
[28] W. Y. Keung and G. Senjanovic, Majorana Neutrinos and the Production of the Right-handed Charged Gauge Boson, Phys. Rev. Lett. 50, 1427 (1983).

[29] A. Datta, M. Guchait, and A. Pilaftsis, Probing lepton number violation via majorana neutrinos at hadron supercolliders, Phys. Rev. D 50, 3195 (1994).

[30] T. Han and B. Zhang, Signatures for Majorana Neutrinos at Hadron Colliders, Phys. Rev. Lett. 97, 171804 (2006).

[31] Z. Si and K. Wang, GeV Majorana neutrinos in top-quark decay at the LHC, Phys. Rev. D 79, 014034 (2009).

[32] A. Atre, T. Han, S. Pascoli, and B. Zhang, The search for heavy Majorana neutrinos, J. High Energy Phys. 05 (2009) 030.

[33] P. Fileviez Perez, T. Han, and T. Li, Testability of type I seesaw at the CERN LHC: Revealing the existence of the B-L symmetry, Phys. Rev. D 80, 073015 (2009).

[34] P. S. B. Dev, A. Pilaftsis, and U. k. Yang, New Production Mechanism for Heavy Neutrinos at the LHC, Phys. Rev. Lett. 112, 081801 (2014).

[35] D. Alva, T. Han, and R. Ruiz, Heavy Majorana neutrinos from $W \gamma$ fusion at hadron colliders, J. High Energy Phys. 02 (2015) 072.

[36] C. Degrande, O. Mattelaer, R. Ruiz, and J. Turner, Fullyautomated precision predictions for heavy neutrino production mechanisms at hadron colliders, Phys. Rev. D 94, 053002 (2016).

[37] S. Antusch, E. Cazzato, and O. Fischer, Sterile neutrino searches at future $e^{-} e^{+}, p p$, and $e^{-} p$ colliders, Int. J. Mod. Phys. A 32, 1750078 (2017).

[38] Y. Cai, T. Han, T. Li, and R. Ruiz, Lepton number violation: Seesaw models and their collider tests, Front. Phys. 6, 40 (2018).

[39] N. Liu, Z. G. Si, L. Wu, H. Zhou, and B. Zhu, Top quark as a probe of heavy Majorana neutrino at the LHC and future colliders, Phys. Rev. D 101, 071701 (2020).

[40] G. Cvetič, C. S. Kim, and J. Zamora-Saá, $C P$ violations in $\pi^{ \pm}$meson decay, J. Phys. G 41, 075004 (2014).

[41] G. Cvetič, C. S. Kim, and J. Zamora-Saá, $C P$ violation in lepton number violating semihadronic decays of $K, D$, $D_{s}, B, B_{c}$, Phys. Rev. D 89, 093012 (2014).

[42] C. O. Dib, M. Campos, and C. S. Kim, $C P$ violation with Majorana neutrinos in $K$ meson decays, J. High Energy Phys. 02 (2015) 108.

[43] G. Cvetic, C. Dib, C. S. Kim, and J. Zamora-Saa, Probing the Majorana neutrinos and their $C P$ violation in decays of charged scalar mesons $\pi, K, D, D_{s}, B, B_{c}$, Symmetry 7, 726 (2015).

[44] G. Cvetic, C. S. Kim, R. Kogerler, and J. Zamora-Saa, Oscillation of heavy sterile neutrino in decay of $B \rightarrow \mu e \pi$, Phys. Rev. D 92, 013015 (2015).

[45] G. Cvetic, C. S. Kim, S. Mendizabal, and J. Zamora-Saa, Exploring $C P$-violation, via heavy neutrino oscillations, in rare B meson decays at Belle II, Eur. Phys. J. C 80, 1052 (2020).

[46] R. M. Godbole, S. P. Maharathy, S. Mandal, M. Mitra, and N. Sinha, Interference effect in LNV and LNC meson decays for left right symmetric model, arXiv:2008.05467.

[47] J. Zhang, T. Wang, G. Li, Y. Jiang, and G. L. Wang, Study of two quasidegenerate heavy sterile neutrinos in rare meson decays, Phys. Rev. D 103, 035015 (2021). 
[48] J. Zamora-Saa, Resonant $C P$ violation in rare $\tau^{ \pm}$decays, J. High Energy Phys. 05 (2017) 110.

[49] S. Tapia and J. Zamora-Saá, Exploring CP-Violating heavy neutrino oscillations in rare tau decays at Belle II, Nucl. Phys. B952, 114936 (2020).

[50] F. Najafi, J. Kumar, and D. London, $C P$ violation in rare lepton-number-violating $W$ decays at the LHC, J. High Energy Phys. 04 (2021) 021.

[51] Z.z. Xing, Correlation between the charged current interactions of light and heavy Majorana neutrinos, Phys. Lett. B 660, 515 (2008).

[52] F. F. Deppisch, P. S. Bhupal Dev, and A. Pilaftsis, Neutrinos and collider physics, New J. Phys. 17, 075019 (2015).

[53] M. Agostini et al. (GERDA Collaboration), Improved Limit on Neutrinoless Double- $\beta$ Decay of ${ }^{76} \mathrm{Ge}$ from GERDA Phase II, Phys. Rev. Lett. 120, 132503 (2018).

[54] S. Pascoli, M. Mitra, and S. Wong, Effect of cancellation in neutrinoless double beta decay, Phys. Rev. D 90, 093005 (2014).

[55] P. Abreu et al. (DELPHI Collaboration), Search for neutral heavy leptons produced in $\mathrm{Z}$ decays, Z. Phys. C 74, 57 (1997); Erratum, Z. Phys. C 75, 580 (1997).

[56] E. Fernandez-Martinez, J. Hernandez-Garcia, and J. LopezPavon, Global constraints on heavy neutrino mixing, J. High Energy Phys. 08 (2016) 033.

[57] A. M. Sirunyan et al. (CMS Collaboration), Search for heavy Majorana neutrinos in same-sign dilepton channels in proton-proton collisions at $\sqrt{s}=13 \mathrm{TeV}$, J. High Energy Phys. 01 (2019) 122.

[58] P. Nason, S. Dawson, and R. K. Ellis, The total cross-section for the production of heavy quarks in hadronic collisions, Nucl. Phys. B303, 607 (1988).
[59] W. Bernreuther, A. Brandenburg, Z. G. Si, and P. Uwer, Top Quark Spin Correlations at Hadron Colliders: Predictions at Next-to-Leading Order QCD, Phys. Rev. Lett. 87, 242002 (2001).

[60] W. Bernreuther, A. Brandenburg, Z. G. Si, and P. Uwer, Top quark pair production and decay at hadron colliders, Nucl. Phys. B690, 81 (2004).

[61] J. Pumplin, D. R. Stump, J. Huston, H. L. Lai, P. M. Nadolsky, and W. K. Tung, New generation of parton distributions with uncertainties from global QCD analysis, J. High Energy Phys. 07 (2002) 012.

[62] G. L. Bayatian et al. (CMS Collaboration), CMS technical design report, volume II: Physics performance, J. Phys. G 34, 995 (2007).

[63] G. Aad et al. (ATLAS Collaboration), Expected performance of the ATLAS experiment-Detector, trigger and physics, arXiv:0901.0512.

[64] J. Alwall, R. Frederix, S. Frixione, V. Hirschi, F. Maltoni, O. Mattelaer, H. S. Shao, T. Stelzer, P. Torrielli, and M. Zaro, The automated computation of tree-level and next-toleading order differential cross sections, and their matching to parton shower simulations, J. High Energy Phys. 07 (2014) 079.

[65] T. Sjostrand, S. Mrenna, and P.Z. Skands, PYTHIA6.4 physics and manual, J. High Energy Phys. 05 (2006) 026.

[66] http://conway.physics.ucdavis.edu/research/software/pgs/ pgs4-general.htm.

[67] M. Cacciari, G. P. Salam, and G. Soyez, FastJet user manual, Eur. Phys. J. C 72, 1896 (2012).

[68] M. Cacciari, G. P. Salam, and G. Soyez, The anti- $k_{t}$ jet clustering algorithm, J. High Energy Phys. 04 (2008) 063. 